\title{
Fellowship training in Canada
}

$\mathrm{n}$ this issue of $C 7 S$, we publish an article by Nousiainen and colleagues ${ }^{1}$ that attempts to evaluate surgical fellowship training in Canada. The perceived need for fellowship training has increased. Once a very American concept, more and more fellowships are being offered in Canada. But it seems little exploration has gone into the need for or design of fellowship training. The authors correctly point out that most of our "scientific" knowledge on fellowship training comes from the US experience. What kind of guidance is available from the Royal College of Physicians and Surgeons of Canada? The Royal College has really shown few data to date about what the Canadian experience is or has been, and realistically, it is not currently in its purview to do so. However, the need to seek fellowship training must in some way reflect the existing residency training programs. As such, it only makes sense for the Royal College to in some way examine the needs of fellowship programs.

Certainly, we can all see the need for most academic programs to be staffed by people with the kind of specialized knowledge that comes with fellowship training. But if programs predominantly comprise fellowship-trained educators providing fellow-oriented teaching with the residents, does this model benefit education? Does the fact that a fellow was present on a service mean that the resident's hands-on surgical training was actually decreased? We do not know how current resident training influences what education, if any, they will need as fellows. There is a lack of a Canadian standard operating protocol to deal with this. I think the paper by Nousiainen and colleagues ${ }^{1}$ brings up several concepts and questions, such as those mentioned above, that need to be examined in more detail.
I believe the Royal College has stated its intent to try to clarify the relation between specialized fellowship training and accreditation. But the Royal College processes seem to be in a state of flux: we are constantly changing how exams are given, what metrics are being judged important for training or how postgraduate education should be optimized. How do we integrate the fellowship programs into this overarching training strategy, especially among the diverse surgical training programs? How involved in the policing of the thousands of fellowship training positions across Canada can we realistically expect the Royal College to become? And how much should it cost for such an accreditation process? Should there be any charge if the fellowship programs are so diverse in nature? Therefore, ensuring that fellowship training is adequate and still does not subtract from the residency experience looks daunting. It seems to be a difficult task if we are not only unclear on the target, but also unsure of who actually holds the bow and arrow. Thankfully, I am not being asked to place an apple on my head ... or am I? I'll have to ask our program director to volunteer for that!

\section{Edward J. Harvey, MD \\ Coeditor, Canadian fournal of Surgery}

Competing interests: None declared.

\section{References}

1. Nousiainen MT, Latter DA, Backstein D, et al. Surgical fellowship training in Canada: What is its current status and is improvement required? Can 7 Surg 2012;55:58-65.

DOI: $10.1503 /$ cjs. 034311 